\title{
Promoting knowledge exchange and collaboration on healthy built environment: A new online discussion forum at NCCEH
}

\author{
Tina Chen* \\ Environmental Health \& Knowledge Translation Scientist, \\ National Collaborating Centre for Environmental Health, \\ Vancouver, BC, Canada
}

"Change is inevitable. Growth is optional" - John C. Maxwell, leadership expert, author, speaker.

The built environment is recognized in the Canadian Chronic Disease Indicator Framework as a determinant of chronic diseases and conditions throughout the life course of individuals (Betancourt et al., 2014). In the Framework, chronic diseases and conditions include diabetes, stroke, cardiovascular diseases, cancer, mental health disorders, obesity, and osteoporosis, among others. Research has demonstrated that built environment features have the potential to mitigate the risk factors of several chronic diseases and conditions. Healthy built environment (HBE) features can therefore lead to improved population health outcomes such as better physical and mental health and lower rates of obesity and injuries (BC Centre for Disease Control, 2018; My Health My Community, 2018). With the rising prevalence of chronic diseases and conditions in Canada, HBE presents opportunities for public health action to encourage healthy behaviours towards improving population health outcomes.

The built environment includes natural and man-made components such as homes, neighbourhoods, workplaces, parks or recreation areas, roads, and transportation systems. Across Canada, $\mathrm{HBE}$ is rapidly becoming a priority in many public health agencies as a result of increasing awareness of the physical, mental, and social health impacts of the built environment. The 2017 annual report from Dr. Theresa Tam, Canada's Chief Public Health Officer, focused on how healthy, equitable, and supportive built environments can positively influence human behaviours, experiences, choices, and perceptions (BC Centre for Disease Control, 2018; My Health My Community, 2018; Tam, 2017). Generally, a healthy community consists of several features (BC Centre for Disease Control, 2018; Canadian Institute of Planners, 2018; Government of British Columbia, n.d.):

- well-connected roads and streets that support healthier lifestyles such as walking, biking, and taking public transit;

- higher residential density in compact neighbourhoods;
- mixed residential and business use;

- access to natural green spaces, healthy and affordable food, and healthy and affordable housing options;

- resilient communities that promote social inclusion and equitable access to amenities and businesses; and

- visually pleasing design and inviting aesthetics.

The multifaceted nature of the built environment highlights the complexity of the linkages between built environment features and population health outcomes. Many of these features are outside the purview and jurisdiction of public health practitioners. Without the engagement of key stakeholders in policy, planning, and government, the ability of public health to improve health outcomes through HBE features is limited (Tam, 2017). An intersectoral collaborative approach is therefore needed to advocate for inclusion of health in planning and policy decisions.

\section{The Role of Public Health Inspectors in HBE}

Many public health agencies have implemented various approaches, including working with provincial and municipal partners and stakeholders to advocate for health considerations in policy and planning decisions. In some provinces, public health inspectors and health promoters in regional health authorities take on these responsibilities, whereas other provinces have specialized healthy community or HBE teams in local health units. Most HBE staff provide health consultations and assessments for local municipalities on community and transportation plans. Some health agencies also conduct research projects to collect locally relevant data, to implement innovative initiatives, and to monitor and evaluate processes and outcomes of activities and initiatives to inform planning and policy decisions. Although the structures and approaches may differ across provinces, the objectives are similar.

In the late 19th century, the sanitary movement spearheaded by public health inspectors and medical doctors utilized zoning and planning as tools to eliminate filth and infectious diseases from cities and towns. This effort gave rise to the land use

*Corresponding author: Tina Chen (email: Tina.Chen@bccdc.ca) 
planning profession (Ashton, 1991; Perdue et al., 2003). Today, in most health agencies the core responsibilities of public health inspectors have changed to focus on reducing risks and hazards for infectious diseases in food, water, sewage, and personal service settings. However, as the greatest burden of illness shifts from infectious to chronic diseases, public health inspectors must return to their roots as change agents. Their role needs to evolve in response to the growing threat of chronic diseases and conditions in order to protect health. As part of the public health workforce, public health inspectors interface with many community partners and business operators and may also be the first point of contact for municipal officials and land use planners. Therefore, they are well situated to collaborate with other public health practitioners to educate and advocate for healthy and supportive built environments.

\section{Healthy Built Environment Linkages Toolkit}

To support public health practitioners and community planners in advocating for and creating healthy and supportive built environments, the $\mathrm{BC}$ Centre for Disease Control Population and Public Health team in BC led the development of the Healthy Built Environment Linkages Toolkit (BC Centre for Disease Control, 2018). Currently in its second edition, the Toolkit is an evidence-based resource for health professionals to articulate scientifically sound and credible messages to inform local government policies and decisions. The Toolkit can also be used by stakeholders such as community planners and local government officials to provide the evidence base for healthy policy and planning decisions and best practices.

The Toolkit considers HBE based on five core features: neighbourhood design, transportation networks, natural environments, food systems, and housing.

Within each feature, fact sheets identify the potential health impacts of healthy community planning and design and provide key evidence-based messages for public health professionals and planners when engaging in policy discussions with local governments and other key stakeholders. The Toolkit also provides health evidence diagrams and summaries of research links, which are graphical overviews that highlight where the research evidence is strongest, moderate, or newly emerging. An interactive online Healthy Built Environment Evidence Diagrams tool was also developed as a part of the Toolkit to allow users to interact with research pathways and access source literature.

\section{NCCEH Healthy Built Environment in Canada Online Forum}

Public health approaches to promote and advocate for health considerations in built environment policies and decisions differ across Canada, and results and lessons learned are often siloed. Through consultations across Canada, the NCCEH learned that HBE working groups and $\mathrm{HBE}$ teams do not readily share and exchange knowledge and information with practitioners in other provinces. A central platform for shared learning on how to better support HBEs in urban, suburban, and rural communities and for open discussions on practice-related questions, appears to be lacking.
To help fill this challenging yet important gap, the NCCEH has developed an online discussion forum in collaboration with the BC Centre for Disease Control Population and Public Health (PPH) team. In addition to enabling learning and knowledge exchange, the discussion forum strives to reduce duplication of efforts by facilitating a channel for HBE practitioners to access a broader network of stakeholders and partners for networking and information exchange. The discussion forum also aims to strengthen the capacity of HBE practitioners to address emerging practice issues and practice gaps and to facilitate the identification of opportunities for cross-sector collaboration and knowledge translation toward new policy solutions.

Organized based on the five core HBE features identified in the Toolkit, the discussion forum aims to bring together individuals who are interested or involved in HBE work. The target audiences for this forum include, but are not limited to, the following sectors and stakeholders:

- public health professionals (including EHOs, population/ community health teams, dietitians, MHOs, community health specialists);

- health organizations;

- federal, provincial, and local governments (planning and design, administration, policy, knowledge translators/ brokers);

- academic institutions (researchers, students in masters, $\mathrm{PhD}$, and urban design/urban planning programs); and

- private sector, including developers and consultants.

The success of discussion forums generally relies on active and continuous participation by a large membership base comprised of a wide variety of players and stakeholders. To help stimulate cross-sector learning and discussion and to encourage growth and continuous participation, the forum will feature:

- Monthly live discussions on various HBE topics prefaced by short presentations given by content experts.

- Provincial champions from above-mentioned sectors will assist with promotion of the forum to their contacts and networks and provide regular content posts to encourage discussions.

- NCCEH and BCCDC PPH staff will moderate the forum, coordinate and host monthly expert webinars and discussions, help connect participants to relevant resources, and support the momentum of conversations by posting practice-related questions.

\section{Conclusion}

The Healthy Built Environment in Canada discussion forum is a unique initiative in Canada. With increasing awareness of the physical, mental, and social health impacts of built environment features coupled with the rising prevalence and burden of chronic diseases and conditions, it is timely to create a panCanadian platform to facilitate cross-sector learning and collaboration in HBE work. Through this forum, public health inspectors will have the opportunity to connect with other public health practitioners and cross-sector partners to better understand built environment concepts, to identify opportunities for the evolution and growth of their roles as a part of the public 
health workforce, and to strengthen their capacity to respond to the rising burden of chronic diseases and conditions.

To apply John C. Maxwell's words in a public health context, changes in disease prevalence are inevitable. How the role of health protection evolves and changes in response to this emerging challenge requires innovation and inspiration garnered through collaborative learning and exchange.

To learn more about and to participate in the discussion forum, please contact Tina.Chen@bccdc.ca. We welcome your feedback to help improve our program implementation and evaluation.

\section{Additional Reading}

- BCCDC healthy built environment linkages toolkit

- Chief public health officer's report on the state of public health in Canada 2017: Designing healthy living

- Canadian Institute of Planners healthy communities committee resources

- NCCEH Built Environment topic page (coming soon to http://www.ncceh.ca/environmental-health-in-canada/ health-agency-projects/built-environment)

\section{References}

Ashton, J. 1991. Sanitarian becomes ecologist: The new environmental health. Br Med J. 302: 189-90. doi: 10.1136/bmj.302.6770.189
BC Centre for Disease Control. 2018. Healthy built environment linkages toolkit: Making the links between design, planning and health, Version 2.0. Vancouver, BC: BC Provincial Health Services Authority.

Betancourt, M. T., Roberts, K. C., Bennett, T.-L., Driscoll, E. R., Jayaraman, G., \& Pelletier, L. 2014. Monitoring chronic diseases in Canada: The chronic disease indicator framework. Chronic Dis Inj Can. 34: 1-30.

Canadian Institute of Planners. 2018. The Canadian Institute of Planners' Draft Policy Statement on Healthy Communities 2018. Available at: http://www.cip-icu.ca/Files/Policies/policy-healthydraft-eng.aspx

Government of British Columbia. n.d. Healthy built environments. Available at: https://www2.gov.bc.ca/gov/content/health/keeping-bc-healthy-safe/healthy-communities/healthy-builtenvironments

My Health My Community. 2018. Social connection and health. Available at: https://www.myhealthmycommunity.org/Portals/0/ Documents/Reports/MHMC_SocialConnections_web.pdf

Perdue, W., Gostin, L., \& Stone, L. 2003. Public health and the built environment: Historical, empirical, and theoretical foundations for an expanded role. J Law Med Ethics. 31(4): 557-566. doi: 10.1111/j.1748-720X.2003.tb00123.x

Tam, T. 2017. The Chief Public Health Officer's Report on the State of Public Health in Canada 2017: Designing healthy living. Ottawa, ON: Public Health Agency of Canada; 2017. 